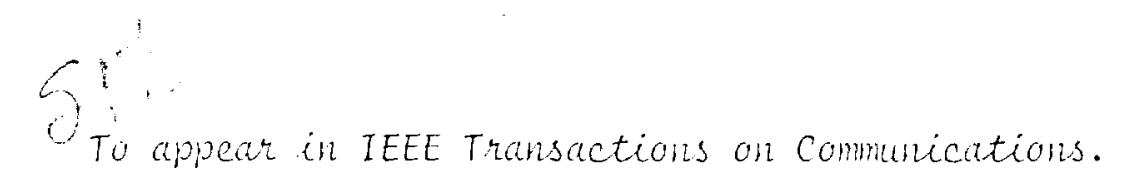

\title{
A Robust Coding Scheme for Packet Video
}

\author{
Y.C. Chen, K. Sayood and D. J. Nelson \\ Department of Electrical Engincering \\ Center for Communication and Infomation Science \\ University of Nebraska-Lincoln
}

\begin{abstract}
We present a haved packet video coding algorithm based on a progressive transmission scheme. The algorith provides good compression and can hanile signiticant packet loss with graceful degradation in the reconstruction sequence. Siatulation results for various conditions are prescnted.
\end{abstract}

\section{INTRODUCTION}

Due io the rapid evolution in the fields of image processing and networking, video information will be an imponant part of tomorrow's teleconmunication systen. Up to now, vidoo transmission hes been mainiy transported over circuit-switched networks. It is quite likely that packet-switched nctworks will cominate the communications world in the near future. Asynchronous transfer mode (ATM) techingues in broadband-1SDN can provide a llexible, independent and high performance environincet for video communication. Therefore, it is necessary to develop technicucs for video tansrinission over such networks.

The classic approach in circuit switching is to provide a "dedicated path," thus reserving a continuous bandwidth capacity in advance. Any unused bandwidth capacity on the allocated circuit is therefore wasted. Rapidly varying signals, like vidco signals, require too much bandwidth to be accommodated by a standard circuit-switching channel. With a certain amount of capacity assigned to a given source, if the output rate of that source is larger than the channel capacity, quality will be degraded. If the generating rate is less than the available capacity. the excess channel capacity is wasted. The use of packet networks allows for the utilization of channel sharing protocols between independent sources and can improve channel utilization. Another point that strongly favors packet-switched networks is the possibility that the integration of services in a network will be facilitated if all of the signals are separated into packets with the same format.

Some coding schemes which support packet video have been explored. Verbiest and Pinnoo proposed a DPCM-based system which is comprised of an intrafield/interframe predictor, a

This work was supported by a grant from the NASA Goddard Space Flight Center (NAG 5-916)

$$
1 .
$$


nonlinear quantizer, and a variable length coder[1]. Their codec obtains stable picture quality by switching between three different coding modes: intraficld DPCM, interframe DPCM, and no replenishment. Ghanbar has simulated a two-layer conditional replenishment codec with a first layer based on hybrid DCT-DPCM and second layer using DPCM[2]. This scheme generates two type of packets: "guarantecd packets" contain vital information and "enhancement packets" contain "add-on" information. Darragh and Baker presented a sub-band codec which attains a user-prescribed fidclity by allowing the encoder's compression rate to vary[3]. The codec's design is based on an algorithm that allocates distortion among the sub-bands to minimize channel entropy. Kishino et al. describe a layered coding technique using discrete cosinc transfom coding, which is suitable for packet loss compensation[4]. Karlsson and Veltcrli presented a sub-band coder using DPCM with a nonuniform quantizer followed by run-length coding for baseband and PCM with run-length coding for nonbaseband[5]. In this paper, a different coding scheme based on a progressive transmission scheme called Mixture Block Coding with Progressive Transmission (MBCPT) $[6,7]$ is investigated. Unlike those methods mentioned abuve, MBCP $[$ docsn't use decimation and interpolation filters to separate the signals into stib-bands. However, it does have hie attractive property of dealing separately with high ficquency and low frequency infomation. This sopamation is obtained by the use of variable bocksize transfom coding.

This paper is organized as follows. First, some of the important characteristics and requinononts of packet video are discussed. In Section 3, the coding scheme called Mixture Block Coding with Prostessive Transmission (MBCPI) is presented. In Section 4, a network simulator used in testing the scheme is introduced. In Section 5, the simulation results are ciiscussol. Finally, in Section 6 the paper is summarized.

\section{CHARACTERISTICS OF PACKET VIDEO}

The demand for various services, such as telemetry, terminal and computer connections, voice communications, and full-motion high-resolution vidco, along with the wide range of bit rates and holding times they represent, provides an impetus for building a Broadband Integrated Service Digital Network (B-ISDN). B-ISDN is a projected worldwide public telecommunications network that will service a wide range of user needs. The continuing advances in the technology of optical fiber transmission and integrated circuit fabrication have been driving forces to realize B-ISDN. The idca of B-ISDN is to build a complete end-to-end switched digital telecommunication network with broadband channels. Still to be precisely defined by CCITT, with fiber transmission, H4 has an access ratc of about 135 Mbps.

Packet-switched networks have the unique characteristics of dynamic bandwidth allocation for transmission and switching resources, and the elimination of channel structure. They acquire and release bandwidth as needed. Because the video signals vary greatly in bandwidth requirement, it is attractive to utilize a packet-switched network for video coded signals. Allowing the transmission rate to vary, video coding based on packet transmission permits the possibility 
of kecping the picture quality constant, by implementing "bandwidth on demand". There are three main merits when transmitting video packets over a packet-switched network:

1. Improved and consistent image quality: if video signals are transmitted over fixed-rate circuits, there is a need to keep the coded bit rate constant, resulting in image degradation accompanying rapid motion.

2. Multimedia integration: as mentioned above, integrated broadband services can be provided using unificd protocols.

3. Improved transmission efficicncy: using variable bit-rate coding and channcl sharing aniong multiple video sources, scenes can be transmitted without distortion if other sources, at the same time, are without rapid motion.

Howcver video transmission over packet networks also has the following drawbacks:

1. The time taken to transmit a packet of data may change from time to time.

2. Pukets may be delayed to the point where, because of constraints due to the Iluman Wual Systom, they have to be discarded.

3. Heaters of packets may be changed because of crrors and delivered to the wrong receiver.

It hes to lie comphasized that the delay/ost effect can reach very high levels if the combined uis:s' requirement excects the acquirable bandwidth and may seriously damage the quality of the image.

When the signals transmitted in the network are nonstationary and circuit-switching is used with imied bandwidth, a buffer betwecn the coder and the channel is needed to smooth out the varying rate. If the amount of data in the buffer cxceds a certain thrchold, the cricoder is instructed to switch into a coding mode that has lower rate but worse quality to avoid buffer overllow. In packet-switched networks, Asynchronous 'Time Division Multiplexing (ATDM) can cficientiy absorb temporal variations of the bit-rate of individual sources by smoothing out the nggregate ce several independent streams in the common network buffers[8].

To scliver packets in a limited time and provide a real time service is a difficult resource allocation and control problem, especially when the scurce generates a high and greatly varying rate. In packet-switched networks, packet losses are inevitable, but use of a packet-switched network yields a better utilization of channel capacity. However, it should be noted that the varying rate requirements of the video coder may not be synchronized with the variations in available channel capacity which changes depending on the traffic in the network. Therefore, the interactions between the coder and the network have to be considered and be incorporated into the requirements for the coder. These requirements include:

1. Adaptability of the coding scheme: The video source we are dealing with has a varying information rate. So it is expected that the encoder should generate different bit rates by removing the redundancy. When the video is still, there is no necd to transmit anything.

2. Insensitivity to error: The coding scheme has to $b c$ robust to the packet loss so that the quality of the image is never seriously damaged. Remember that retransmission is impossible because of the tight timing requirement. 
3. Resy'nchronization of the vidco: Because the varying packet-generating rate and the lack of a common clock between the coder and the decoder, we have to find a way to reconstruct the received data which is synchronous to the display terminal.

4. Control of coding rate: Sensing the heavy traffic in the network, the coding scheme is required to adjust the coding rate by itself. In the case of a congested network, the cocier could be switched to another mode which generates fewer bits with a minimal degradation of image quality.

5. Parallel architecture: The coder should preferably be implemented in parallel. That allows the coding procedure to be run at a lower rate in many parallel streams.

In the rext section, we investigate a coding scheme to see how well it satisfies the above requiromants.

\section{MIXTURE BLOCK CODING WITH PROGRESSIVE TRANS.IISSION}

Minin block Cojing (MBC) is a variable-blocksize transfom coding algorithin which codes tha me with different blocksizes depending upon the complexity of that block area. Low-Comexity areas are coded with a large blocksize transfonn coder while high-complexity wions a.-. coded with snall blocksize. The complexity of the specific block is detemined by the disoricn between the coded and original image when the sime number of bits are used to wete cacin Llock. A more complex image block has higher distortion. The advantage of using $\triangle 1 B C$ in that it does not process different complex regions with the same blocksize. That means $M B B C$ has the ability to choose a finer or coarser coding scheme to deal with different complex parts cit the same image. With the same rate, $\mathrm{MBC}$ is able to provide an image of higher quality than a ceding scheme which codes different complex regions with the same blocksize coder.

When using $M B C$, the image is divided into maximum blocksize blocks. After coding, the distortica between the reconstructed and original block is calculated. The block being processed is subdivided into smaller blocks if that distortion fails to meet the predetermined threshold. The coding-testing procedure continues until the distortion is small cnough or the smallest blocksize is reached. In this scheme, every block is coded until the reconstructed image is satisfactory and then moves to the next block.

Mixture Block Coding with progressive transmission (MBCPT) is a coding scheme which combines $\mathrm{MBC}$ and progressive coding. Progressive coding is an approach that allows an initial image to te transmitted at a lower bit rate which can later be updated[9]. In this way, successive approximations converge to the target image with the first approximation carrying the "most" information and the following approximations enhancing it. The process is like focusing a lens, where the entire image is transformed from low-quality into high-quality. In progressive coding, every pixel value, or the information contained in it, is possibly coded more than once and the total bit rate may increase due to different coding scheme and quality desired. Because only the 
gross features of an image are being coded and transmitted in the first pass, the processing time is greatly reduced for the first pass and a coarse version of the image can be displayed without significant delay. It has been shown that it is perceptually useful to get a crude image in a short time, rather than waiting a long time to get a clear complete image.

With different stopping criterion, progressive coding is suitable for dynamic channcl capacity allocation. If a predetermined distorion threshold is met, processing is stopped and no more refining action is necded. The threshold value can be adjusted according to the traffic condition in the channel. Successive approximations (or iterations) are sent through the channel in progressive coding and lead the recciver to the desired image. If these successive approximations are marked with decreasing priority, then a sudden decrease in channel capacity may only cause the received image to suffer from quality degradation rather than total loss of parts of the images.

MBCPT is a multipass scheme in which cach pass deals with different blocksizes. The first pass codes the image with maximum blocksize and transmits it immediatcly. Only those blocks which fail to meet the distortion threshold go down to the second pass which processes the difference image block, coming from the original and coded innge obtained in the first pass, wh smaller blocks. The difference image coding scheme continucs until the final pass which deals withe minimum size block. At the recoiving end, a crede inage is obtained from the first paes in a short time and the data from following passes serve to cnhance it. Fig. 1 shows the structue of pass consisting of $16 \times 16$ blocks for MBCPT. Fig. 2 shows the parallel structure of MBCET. Coding algorihns using quad trecs have also been proposed by Dreizen[10] and Vaisey and Gersho[11]. In the quad tree coding structure of this paper, the $16 \times 16$ block is coded and the disturion of the block is calculated. If the distortion is greater than the predetermined Wrestuld for $16 \times 16$ blocks, the block is divided into four $8 \times 8$ blocks for additional coding. This coding-cheching procedure is continued until the only image blocks not meeting the threshold are those of size $2 \times 2$. Figure 3 shows the algorithm.

The block size used in the coding scheme should be small enough for ease of processing and storage requirements, but large chough to limit the inter-block redundancy[12]. Larger block size results in higher compression, but it is very difficult to build real-tine hardware for blocksizes larger than $16 \times 16$ because of the increase in the number of computations. So, $16 \times 16$ is chosen to $b e$ the largest blocksize. The minimum blocksize detemines the finest visual quality that is achicvable in the busy arca. If the minimum blocksize is too large, it is possible to observe the blockiness in the coded edge of spherical objects because the coding block is square. In order to match the zonal transform coding used in this paper, $2 \times 2$ is the smallest blocksize and there are four passes $(16 \times 16,8 \times 8,4 \times 4,2 \times 2)$ in this scheme. Fig. 4-7 show images from the 4 passes.

After applying the discrete cosine transform, only four coefficients, including the $\mathrm{dc}$ and three lowest order frequency coefficients, are coded and others are set to zero. The de coefficient in the first pass is coded with an 8-bit uniform quantizer due to the fact that it closely reflects the average gray level for that image block and is hard to model. The dc coefficient in the subscquent passes follows a laplacian model, and a 5-bit optimal laplacian nonuniform quantizer is used to also follow a laplacian model with a variance greater than that of the de coefficient and can thercfore also be coded using a laplacian quantizer. As an altemative, an LBG vector quantizer with a 512 codebook size is used to quantize the vector which comprises the three ac 
coefficients. The initial threshold of each pass is selected beforchand and is readjustable during the operation according to the channel condition and quality required.

Because only partial blocks which fail to mect the distortion threshold need to be coded, side infomation is needed to instruct the receiver on how to reconstruct the image. One bit of overhead is needed for each block. If a block is to be divided, a 1 is assigned to be its overhead; if not, a 0 is assigned. The example shown in Fig. 8 has the following overhead: $1,1001,1001,1001,1001,1001$.

The interframe coder used in this paper is a differential scheme which is bascd on MBCPT. This coder processes the difference image coming from the current frame and the previous frame which is locally decoded from the first three pass data. Fig. 9 shows the algorithm of this coder. Fig. 10 shows a different scheme which does the local decoding with all four passes. Fron F;g. 11, it can be seen that when there is no packet loss, the performances of these wo schemes are quite the same. But when congestion occurs in the network, with the priorities asigned to packets, packets from pass 4 are expected to be discarded first. In this case, the perfomance (from Fig. 12) of the scheme in Fig. 9 is much better than the one in Fig. 10. Therefore the coding scheme in Fig. 9 is used in our simulation. In this paper, the Kronkite motion sequence from the USC database with 16 frames is used as the simulation source. Livy image is $256 \times 256$ pixels wih graylcvels ranging from 0 to 255 . It is similar to a viteo confercacing type image which has neither rapid motion nor scenes changes. Due to this characteristic, advanced techniques like motion detection or motion compensation have not becn usc. but could be implemented when broadcasting video.

From the datastrean output that is listed in Table 1, we can see that the data in pass 4 represents $30-40 \%$ of the entire data. This part of the data is involved in increasing the sharpness of the imese and is usually labeled with the lowest priority in network. We therefore call this the least significant pass(LSP). Wiuh a substantial possibility of being discarded due to low priority, those packets from pass 4 won't be used to reconstruct the locally decoded image and be storcd in the frame memory. This prevents the packet loss crror propagating into following frames if the lost packet bclongs to pass 4 .

\section{SIMULATION NETWORK}

The network simulator used for this study was a modified version of an existing simulator developed by Nelson et al.[13]. A brief description of the simulator is provided here.

\section{A. Introduction}

As mentioned in section 2, tomorrow's integrated telecommunication network is a very complicated and dynamic structure. Its efficiency requires sophisticated monitoring and control algorithms with communication between nodes reflecting the existing capacity and reliability of system components. The scheme for communicating information regarding the operating status 
is called the system protocol. Since the communication of system information must flow through the channel, it reduces the overall capacity of the physical layers, but hopefully provides a more efficient system overall. Therefore, system efficiency depends entirely upon these protocols, which, in tum, depend upon the system topology, communication channel properties, nodal memory and component reliability. Most network protocols have been developed to provide high reliability in topological structures with reasonably high channel reliability.

In order to fit into the purpose of this study, most modifications which were made to the simulator were in those modules concerning the network layer. Since the simulator is structured in modules which represent, to some degree, the ISO Model for packet switched networks, a more detailed description about the network layer modules follows.

\section{B. The Network Layer and Basic Operation}

The simulation of a layer at each node is represented by a "proccssor" and one or more "packet queles." All crents are scheduled through the "Sim_Q" which drives the simulator. Initially, the processors are all idle, the packet queucs are all empty and the only tasks scheduled ate tie arival of messages at the various nodes. The simulator operation occurs by cxamining the next event and performing the task indicated. The task may result in the scheduling of aditional crents, generally referred to as task completion times. When a message or packet is placed in the input queue at a node for a given layer, the processor for that queue is marked as busy, the packet is removed from the queuc, and the task to be performed by the processor is schedied for completion. When the task is completed (as a result of the simulator reaching that point in time), the "processor" examines the queve. If the queue is empty, the processor is set idle; otherwise it removes the next message or packet from the queue and schedules the completion of the operation which must be performed. The lajers in the simulator are quite close in operation to the ISO transport, network and datalink layers.

\section{(1) The Session Layer}

In the OSI model, the session layer (SL) allows users to establish "sessions" on local or remote systems. In the simulator, as mentioned above, it contains a relatively simple model of the subscribers, participates in flow-control, and acts as a statistics collector for messages arriving and delivered. At message arrival time (from Sim_Q), the session layer gencrates the "message" with all of its randomly selected attributes and if flow control or node hold-down are not in effect, submits it to the transport layer. It then schedules the next message arrival time. During initialization, the task "SL_Rcv_Msg" for each node is queued in Sim_Q for the arrival time of the first message at that node. When this task is executed by the simulator, a message packet is generated and placed in the transport queue. The arrival of the next message is then queued in Sim_Q with the same task and with an arrival time determined by the random number generator (Poisson Distributed). The only other task performed by the session layer is the "SL_Snd_Msg" task that simulates delivery of messages to the subscribers, develops message statistics and "cleans up" the queues for messages delivered. 


\section{(2) The Transpori Layer}

The basic function of the transport layer at the sending end is to receive the message from the session layer, place it in packets and pass the packets on to the network layer. At the receiving end, the packets are reassembled into a message for delivery to the session layer. To accomplish the complex task of assuring reliable delivery, there is a transport time-out mechanism at both the sending and receiving nodes and a message acknowledgement packet that is sent to the sending node when all packets for the message have been satisfactorily received. At the sending end, if a message acknowledgment is not received in the allotted time period, the message can be retransmitted. In the simulations reported in this paper, the retransmission feature was not used. At the receiving end, if all packets are not received in the specified period of time, the entire message is discarded. It is recognized that in some nctworks, packetization takes place at the network level, leaving the transport layer responsible only for message-level structures. Reassently, depending upon the protocol, can take place as low as the datalink level. These tasks were both placed in the transport layer, but are modular, and could be cxtracted and placed cisewhere. Also, the simulator was originally designed for datagram service, and since the packets do not nocessarily arrive in order, it is unlikely that assembly would take place at the datalink lovel.

\section{(3) The Network Layer}

The network layer is concemed with controlling the operation of the network. A key design issue is detemining how packets are routed from source to destination. Another issue is how to aroid the congestion caused when too many packets are presented to the network at the same time. In the simulator, the network layer performs all of the functions related to these two aspects with the cxception of that aspect of flow control which takes place at the session layer, and the recovery protocols which require some service from the datalink layer. It also activates new channels when needed and determines when packets originating at other nodes are to be discarded. The network layer is currently the most dynamic with regard to the coding of modules. Five modules currenty comprise the network layer. These include relatively static modules; one module for capturing lines or channcls when more capacity is required and releasing them when they are rot necied; one module for the retwork processor and queue handling and one module for the routines which are common to most routing algorithms. This leaves two modules for the dynamic parts of the routing and flow control algorithms.

\section{(4) The Datalink Layer}

The main task of the datalink layer is to take a raw transmission facility and transfom it into a line or channel that appears free of transmission errors to the network layer. It simulates the sending of the message over the channel and the delivery at the other end. When a packet is received, the datalink acknowledgement is initiated either by the piggy-back acknowledgement or by generating a datalink acknowledgement packet. As mentioned previously, the datalink level also simulates the physical layer on a statistical basis. (Entered bit error rates are used in conjunction with a random number generator to determine if messages are corrupted.) When a line is "brought up", health packets are used to establish initial connections. Also, when a 
line "gocs down", an active node will immediately issue health check packets to ascertain when the channel is again available.

\section{Modifications}

A major problem of using this system as a simulation tool for the study of packet video is that as initially designed the system did not actually transmit messages from node to node. While a "packet" carrying all the necessary describing information moved from node to node, there no actual data in the packet. Therefore, modifications had to be made to the simulator to accommodate the vidco data. In the sending node, a field called "Image" which contains real image data is attached to the record "Packet_Ptr" allocated to the message generated in the scssion layer. There are three new modules in this layer. First, "Get_Inage" puts the image bata into the image field of a message generated at a specific time and node. Second, "linage_writable" checks to see if there is any image data that still needs to be transmited. If that is tre, the following message, generated at that specific node, is still the image message and contains sone image data. Third, "Receive_Image" collects the image data in the session layer of the reciving node when the flag "Image_Complete" is on. In module "Session_Msg_Arive", difievent pricritics are assigned to difierent messages. In module "Scssion_.Msg_ Send", some statistics are calculated including the number of lost image packets and the transmission delay for image packets.

In tie orginal deisgn, the transport layer simply duplicated the same packet with different assigned secuential packet numbers without actually packetizing the message. The module "Transpor Packetize" has been modificd to really packetize the image data which resides in the message record queued in "Transport_Q" when it is called. The module "Transport_Reassemble" is called to reassemble these image packets according to their packet number when the flag "Image_Content" defined in "Packet_Ptr" is true. The network layer is responsible for routing and fow-control. This module was already very well developed, so the modifications to be performed liere were relatively minor. In the datalink layer, in order to simulate the delivery of packets dirough the channel, a new packet is generated at the receiving node and the information including the image data from the transmitted packet (which will still be resident at the sending node) are copied into it. Using existing bit-error-rates, the transmission success rate can be set and bit errors can be inserted in both the data and control bits in the packet. Errors in the control bits are simulated separately as long as the error rates are consistent. If an error in control bits occurs, the transmission is assumed to fail and retransmission will occur, again depending on the threshold of the timeout number. In addition to the modifications made to the layer modules, we had to arrange some new memory elements allocated for image messages and packets. In order to make sure the simulation is run in the steady state, the image data is made available to the network after some simulation time has passed. 


\section{INTERACTION OF THE COUER AND THE NETWORK}

When the video data is packed and sent into a nonideal network, some problems cmerge. These are discussed in the following section.

\section{A. Packetization}

The task of the packetizer is to assemble video information, coding mode information, if it exists, and synchronization information into transmission cells. In order to prevent the propagation of the error resulting from the packet loss, packets are made independent of each other and no data from the same block or same frame is separated into different packets. The segmentation process in the transport layer has no information regarding the video fomat. To avoid the bit stream being cut randomly, the packetization process has to be integrated with the encoder, which is in the presentation layer of the user's premise. Otherwise, some overhead has 10 be aded into the datastream to guide the transport liayer to perfom the packetization in the desind momer. In order to limit the delay of packetization, it is necessary to stuff the last cell of a pactet video wihl dummy bits if the cell is not completely full.

Evry pecket must contain an absolute address which indicates the location of the first block it carries. Because every block in MBCPT has the same number of bits in cach pass, there is no need to iteicate the relative address of the following blocks contained in the same packet. There abuys cxisis a tradeoff between packaging efficiency and error resilicnec. If cror resilience is coiliberble, one packet should contain a smaller number of blocks. However, since each chamel access by a station contains overhead, the packet length should be large for transmission cficioncy. Fixed length packetization is used in this paper for simplicity.

Becuse of the structure of the coding scheme, the packets are classified into four priorities, with the packets from the first pass classificd as the highest priority packets, and the packets from the fourth pass as the lowest priority packets.

This priority assigmment also reflects the importance of the various packets to the reconstriction of the image sequence at the receiver. Table 1 shows the effect of approximately the same amount of packets lost in each pass on the reconstructed error in the received sequence.

\section{B. Error Recovery}

There is no way to guarantee that packets won't get lost after being sent into the network. Packet loss can be mainly attributed to two problems. First, bit errors can occur in the address ficld, leading the packets astray in the network. Second, congestion can exceed the networks management ability and packets are forced to be discarded due to buffer overflow. Effects created by higher pass packet loss (like pass 4 ) in MBCPT coding will be masked by the basic passes and replaced with zeros. The distortion is almost invisible when viewing at video rates because the lost area is scattered spatially and over time. However, low pass packets loss (like pass 1), though rare due to high priority, will create an erasure effect due to packetization and the effect is very objectionable. 
Considering the tight time constraint, retransmission is not feasible in packet video. It may also result in more severe congestion. Thus, error recovery has to be performed by the decoder alone. In our differential MBCPT scheme, the packets from pass 4 are labeled lowest priority and form a great part of the complete data. These packets can be discarded whenever netwoik congestion occurs. That will reduce the network congestion and won't cause too much degradation in quality. The erasures caused by basic pass loss are simply covered with the reconstructed yalues from the corresponding area in the previous frame. This remedy secms insufficient even when there is only small amount of motion in that area. Motion detection and motion compensation could be used to find a best matched area for replacement in the previous frame.

Sise iniomation in the MBCPT decoding scheme is very important. So, this vital information is not allowied to get lost. Two methods can be used for protection. First, error control coding, like block coles or convolutional codes, can be applicd in both directions along with and perpendicular to the packetization. The former is for bit cror in the data field while the laticr is for pacte loss. The minimum distance that the error control coding should provide depencis on the rework's probability of packet loss, correlation of such loss and channel bit error ratc.

Second, from rable 2, we can sce that the output rate of side information and pass 1 and cyen pass 2 is quite steady. It secms feasible to reserve a certain amount of channel capacity 10 these oumes to cnsure their timely arrival. That means circuit-switching can be used for imporan and stendy cata.

\section{Flow Control}

In cret to shicld the viewer from severe network congestion, there are some flow control schemes which are considered useful. If there is an interaction between the encoder and the transport layer, then the encoder can be informed about the network condition. Depending on that, the encoder can adjust its coding scheme. In the MBCPT coding scheme, if the buffer is getting full, that means that the bit generating rate is overwhelming the packetization rate and the cncoder will switch to a coarse quantizer will fewer steps or loosens the threshold to decrease its output rate. In this way, smooth quality degradation is obtainable. However, this also complicates the encoder design.

It is possible to use the congestion control of the network protocols to prevent the drastic quality change by assigning different priorities to packets from different passes. Without idcntifying the importance of each packet and discarding packets blindly sometimes brings disaster and can cause a session shut down. For example, if the side information gets lost it can have a severe impact on the decoding process. In the MBCPT coding scheme, side information and packets from pass 1 are assigned highest priority and higher pass packets are assigned with decreasing priority. 


\section{Interaction with Protocols}

In the ISO model, physical, datalink and network layers comprise the lower layers which form a nctwork node. The higher layers have uransport, session, presentation and application layers and typically reside in a customer's premises. The lower layers have to do nothing about the signal processing and only work as a "packet pipe". The physical layer requires adequate capacity and low bit-crror-rate which are determined only by technology. The datalink layer can only deal with link-management because all the mechanics, like requesting retransmission, is not feasible in packet video transmission. The network layer has to maintain orderly transmission by deleting the delay jitter with input buffering. Otherwisc, it can take care the network congestion by assigning transmission priority.

As the higher layers reside in the custoner's premises, it performs all the functions of the packet viceo coder. The transport lajer docs the packetization and reassembly. The packet length can be fixed or variable. Fixed packet length simplifics segmentation and packet handling while a vatable pecket length can kecp the packetization delay constant. The sessicn layer supervises set-up and tear-down for sessions which have different types and quality. There is always a tradcolf between quality and cost. The quality of a set-up session can be decmined by the thestold in the coding scheme and the prority assignment for transmission. Of course, the bolke the quality, the higher the cost. Fig. 13 shows the tradeoff between PSNR and video cutput rate by ajusting thresholds. The presentation layer does most of the signal processing, including seraration and compression. Because it knows the video fomat exactly, if any ertor concalment is required, it will be perfomed here. The application layer works as a boundary between the user and the network and deals with all the analog-digital signal conversion.

\section{PERFORMANCE RESULTS}

Results obtained in this packet video simulation show that substantial compression can be obtaincd while mainaining high image quality through the use of this differential MBCPT scheme. The monochrome sequence used in this simulation contains 16 frames, each of size $256 \times 256$ pixels with 8 bits per pixel, which results in a bit rate of $15.3 \mathrm{Mbits} / \mathrm{s}$, given a video rate of $30 \mathrm{frames} / \mathrm{s}$. As Tablc 2 shows, the average data rates of our system is $1.539 \mathrm{Mbits} / \mathrm{s}$. The compression rate is about 10 with a mean PSNR of $38.74 \mathrm{~dB}$ where PSNR is defined as

PSNR $=\log _{10} \frac{\sum(255)^{2}}{\sum\left(x_{i j}-\hat{x}_{i j}\right)^{2}}$

Fig. 14 shows the data rate of the sequence frames with side information, 4 passes and total rate. It is clear that the data rate of pass 1 is constant as long as the quantization mode remains the same. Side infornation and data from pass 2 , even pass 3 , is also. The data rate of pass 4 is bursty and highly. uncorrelated. As pass 4 data is not essential to the reconstruction of the image, the rate profiles as shown in Figure 14 and Table 1 suggest the use of a reserved channel of some sort for passes 1-3 and the side information, and a perhaps more unreliable channcl for pass 4 data which comprises more than $30 \%$ of the total traffic. Such a situation 
can be accommodated in a variety of systems such as a token ring network or a circuit switched network with a packet-switched overlay.

Fig. 15 shows the PSNR for each frame in the sequence. Notice that the standard deviation of the PSNR is only $0.2 \mathrm{~dB}$, which implics a substantial uniformity of quality, at least in tcrms of objective performance measures. If constancy with regards to some subjective criterion is desired, it would be necessary to incorporate this in the detemination of the thresholds and the decision mechanism for the quad tree. In the simulation, the same threshold has been used throughout the sequence. If further flexibility, say for higher visual quality is desired, a varying threshold can be used for different frames. That may generate a more variable bit rate.

From the difference images of this sequence, frames $1-8$ seem quite motionless while frames 9-13 contain substantial motion. We adjusted the traffic condition of the network to force some of the packets to get lost and thus check the robusticss of the coding scheme. Heavy traffic was set up in the motionless and motion period scparatcly. The average packet loss percentage was $3.3 \%$ which is considered high for most networks. Fig. 16 shows images which suffered packet losses from pass 4 . As can be secn, the effect of lost packets is not at all severe, even if the lost packet rate is unrealistically high. This is because the performance from the first three passes is relatively good and the packet from the fourth pass is not essential for reconstruction. Fig. 17 shows the case when packet loss occurs in pass 1. Clearly there are visible defects in the motion period. What's worse, the error will propagate to the iollowing frames. Apparently, the replenishing scheme used here is not sufficient in areas with motion. It is believed that this inconsistency can be eliminated with a motion compensator algorithm which would find the approprite area for replenishment and cror concealment which limits the propagation of error.

\section{CONCLUSIONS}

The network simulator was used only as a channcl in this simulation. In fact, before the realtime processor is built, a lot of statistics can be collected from the network simulator to improve upon the coding scheme. These include transmission delays and losses from yarious passes under different network loads. For resynchronization, the delay jitter between received packets can also be estimated from the simulation. The environment for tomorrow's telecommunication has been described and requires a flexibility which is not possible in a circuit-switched network. With all the requirements for applying packet video in mind, MBCPT has been investigated. It is found that MBCPT has appealing properties, like high compression rate with good visual performance, robustness to packet lost, tractable integration with network mechanics and simplicity in parallel implementation. Some additional considerations have been proposed for the entire packet video system, like designing protocols, packetization, error recovery and resynchronization. For fast moving scenes, the differential MBCPT scheme seems insufficient. Motion compensation, error concealment or even attaching function commands into the coding scheme are believed to be useful tools to improve the performance and will be the direction of future research. 


\section{REFERENCES}

[1] W. Verbiest and L. Pinnoo, "A Variable Bit Rate Codec for Asynchronous Transfer Mode Networks," IEEE J. Select. Areas Commun., vol. 7, pp. 761-770, June 1989.

[2] M. Ghanbari, "Two-Layer Coding of Video Signals for VBR Networks," IEEE J. Select. Areas Commun., vol. 7, pp. 771-781, June 1989.

[3] J. Darragh and R. Baker, "Fixed Distortion Subband Coding of Images for Packet-Switched Nctworks," IEEE J. Select. Areas Commun., vol. 7, pp. 789-800, Junc 1989.

[4] F. Kishino, K. Manabe, Y. Hayashi and H. Yasuda, "Variable Bit-Rate Coding of Vidco Signals for ATM Networks," IEEE J. Select. Areas Commun., yol. 7, pp.801-806, June 1989

[5] G. Karlsson and M. Vetterli, "Packet Video and Its Integration into Network Archiecture," IEEE J. Select. Areas Commun., vol. 7, pp. 739-751, Junc 1989.

[6] M. C. Rost, "Data Compression Using Adaptive Transform Coding," Ph.D. dissertation, Liniverity of Nebrastia-Lincoln, 1988.

(7) M. C. Rost and K. Sayood, "A Progressive Data Compression Scheme Based on Adaptive Transom Coding," Proc. 3Ist Midwest Symposimm on Circuits and System, St.Louis, MO, Alg.1989. pp. $912-915$.

[3] 3. Mreginis, D. Anastassiou, P. Sen, G. Karlsson, J. Robbins, "Perfomance Analysis of Statistical Multiplexing for Packet Vidco Sources," Proc. of the Globecom-87, Tokyo, Japan, Noy. 1957, pp. 47.8.1-10.

19] K. Sloar, Jr. and S. Tanimoto, "Progressive Refinement of Raster Scan Images," IEEE Trans. Comint, vol. COM-28, pp. 871-874, Nov. 1979.

[10] H. M. Dreizen, "Content-Driven Progressive Transmission of Grey-Scale Images," IEEE Trans. Conmun, vol. COM-35, pp. 289-296, March 1987.

[11] D. Vaisey and A. Gersho, "Variable Block-Size Coding," Proc. ICASSP, pp. 1051-1054, April 1987.

[12] Y. S. Ho and A. Gersho, "Variable-Rate Multi-Stage Vector Quantization for Image Coding" in Proc. ICASSP, vol. 2, pp. 1156-1159, 1988.

[13] D. Nelson, K. Sayood, G. Ankenman and H. Chang, "Pnetsim System Programmer's Manual," University of Nebraska-Lincoln, Final Report for ARMY Contract DAAB07-85K-K535, Dec. 1986. 


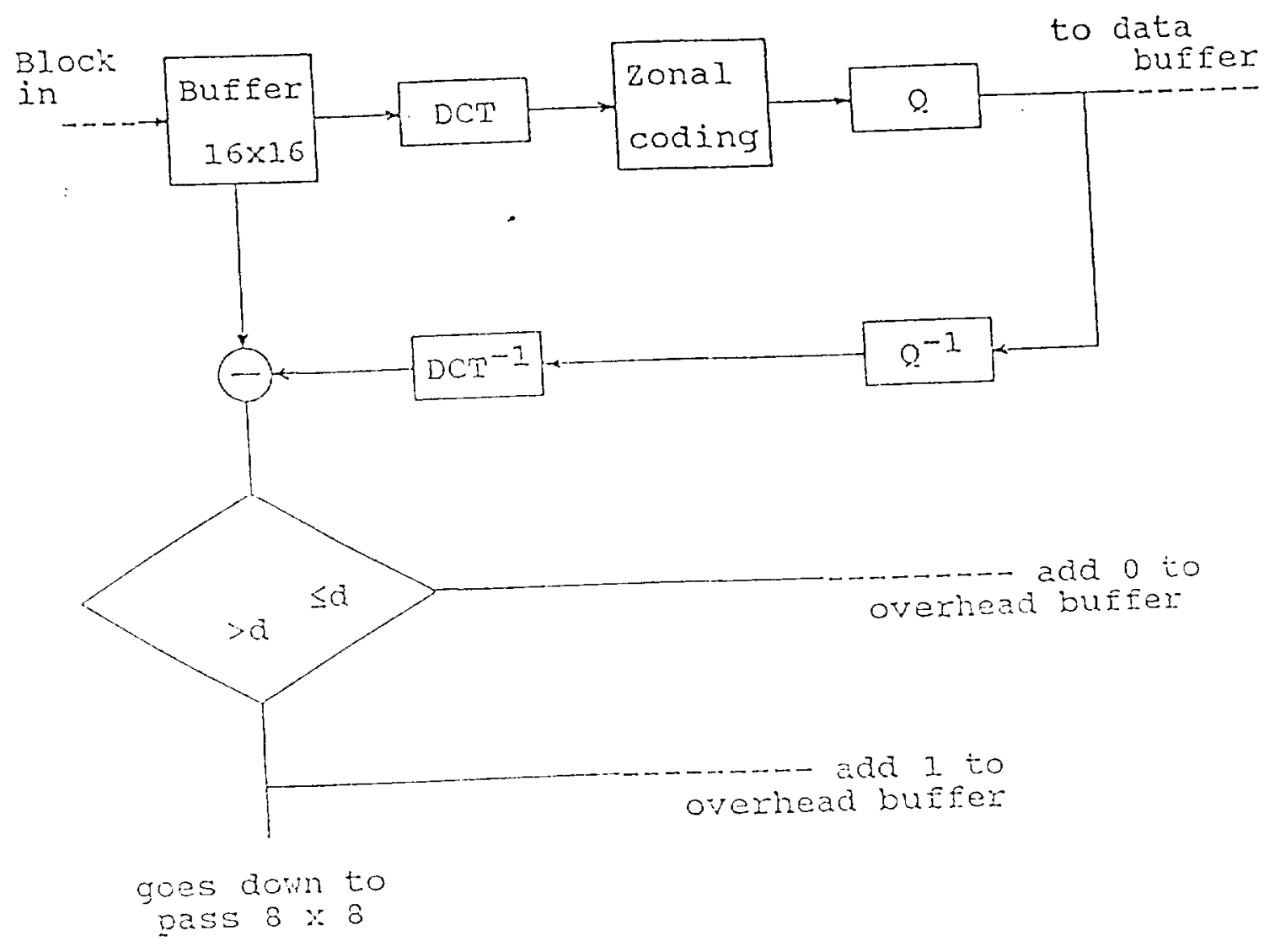

Figure 1 struciure of the first pass consisting of $16 \times 16$ blocks for MBCPT. 


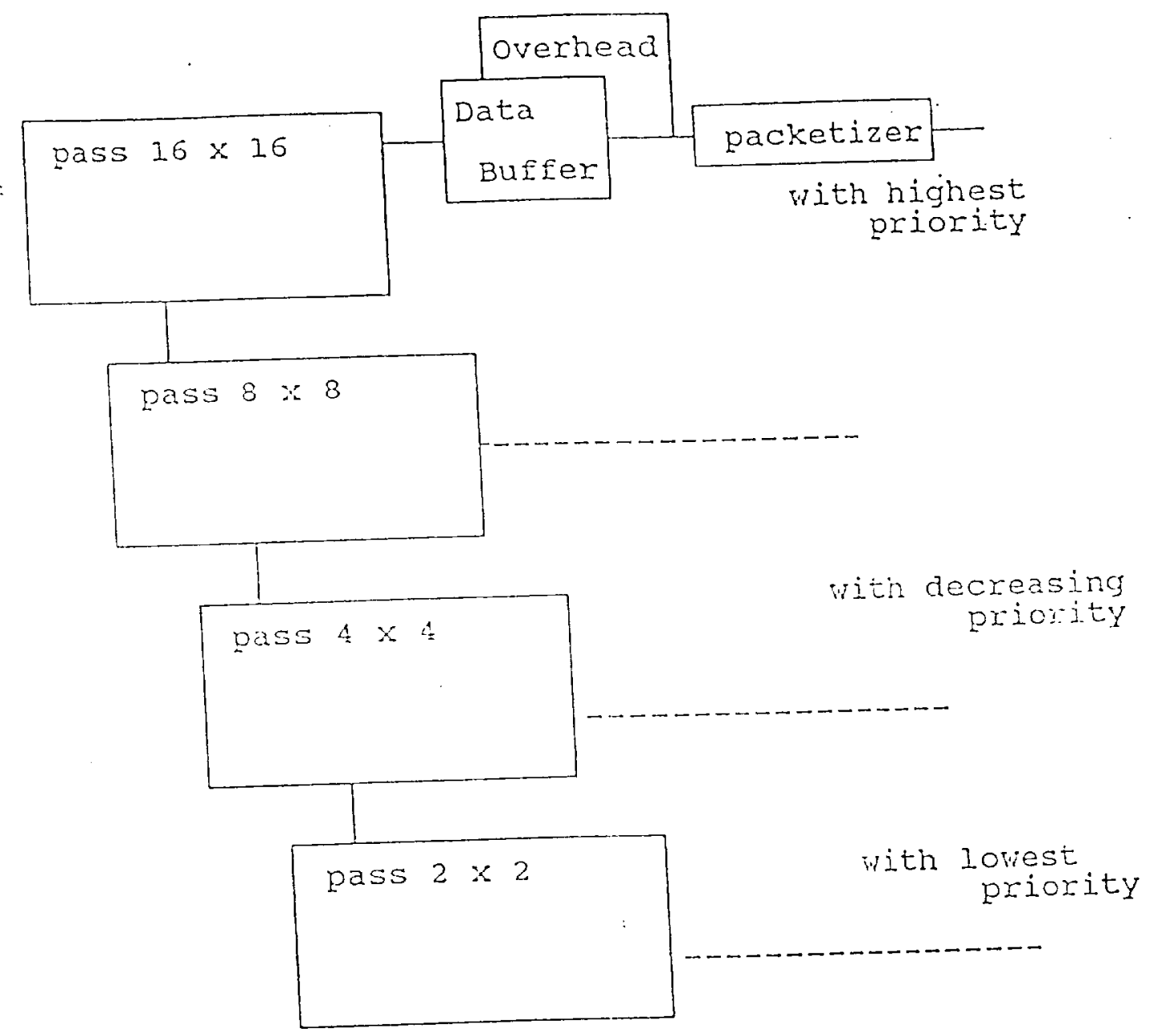

Figure 2 Parallel structure for $\mathrm{BBC} P \mathrm{BT}$. 
$16 \times 16$

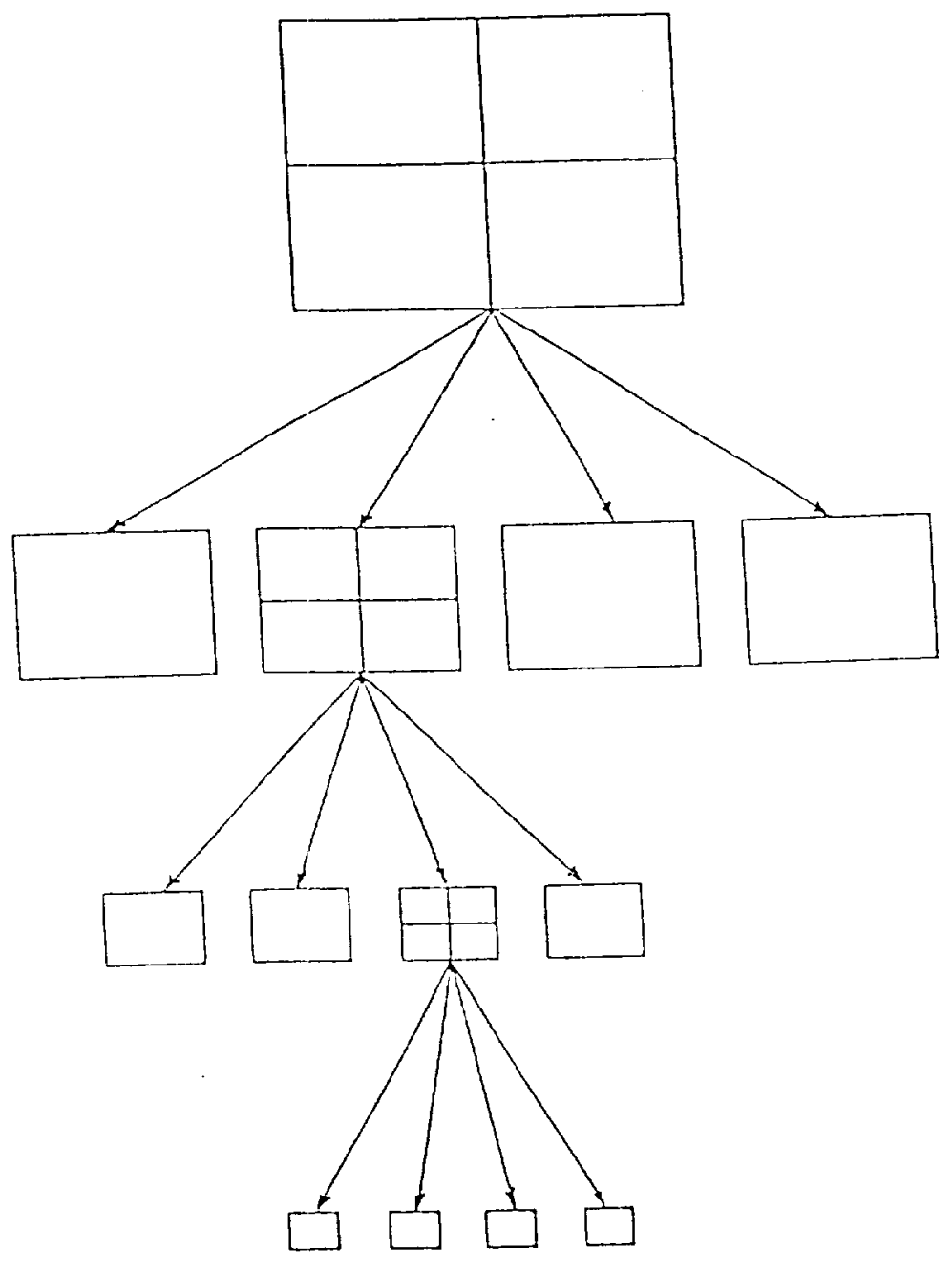

$2 \times 2$

Figure 3 Example of the quad tree structure. 

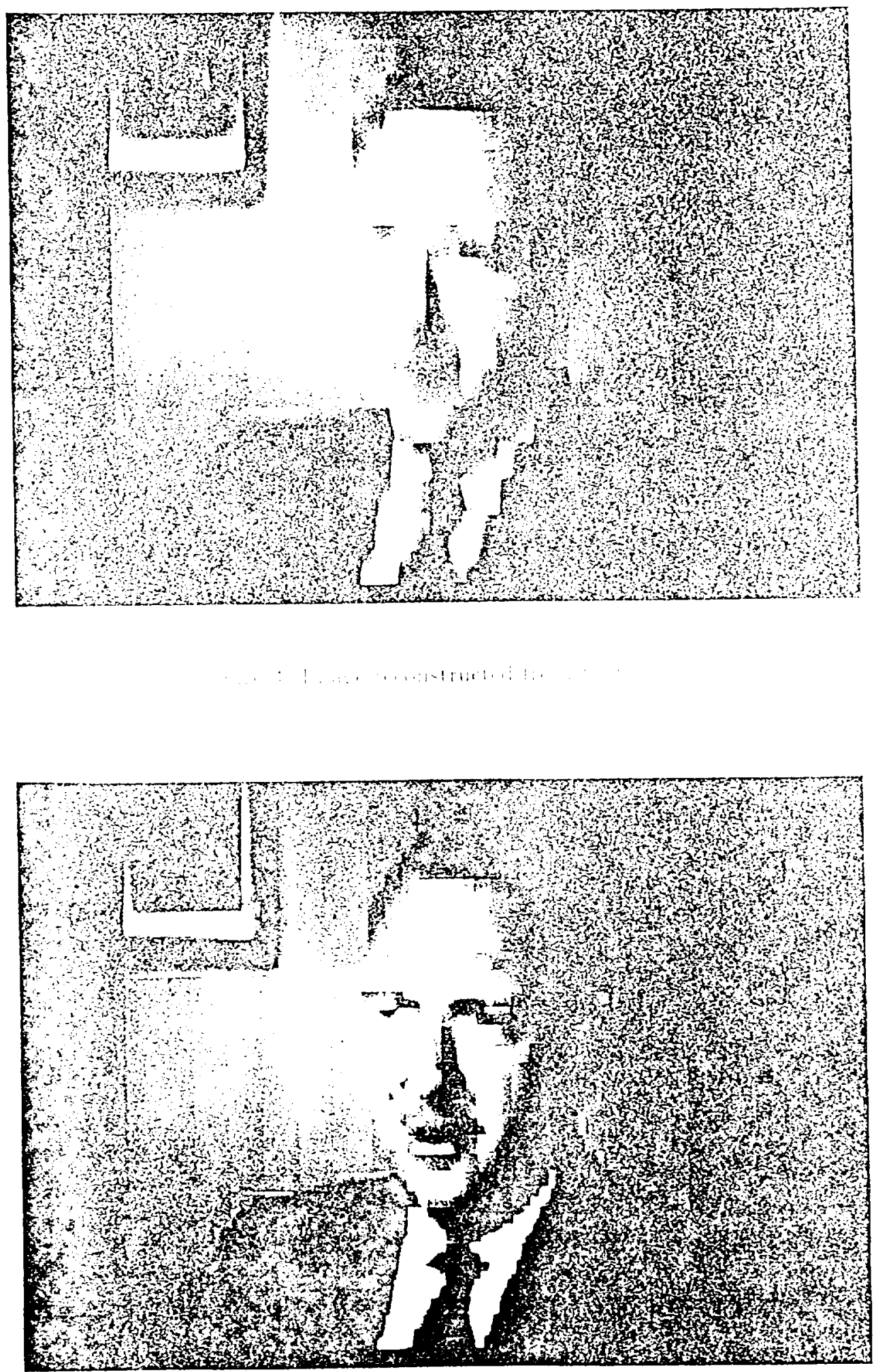

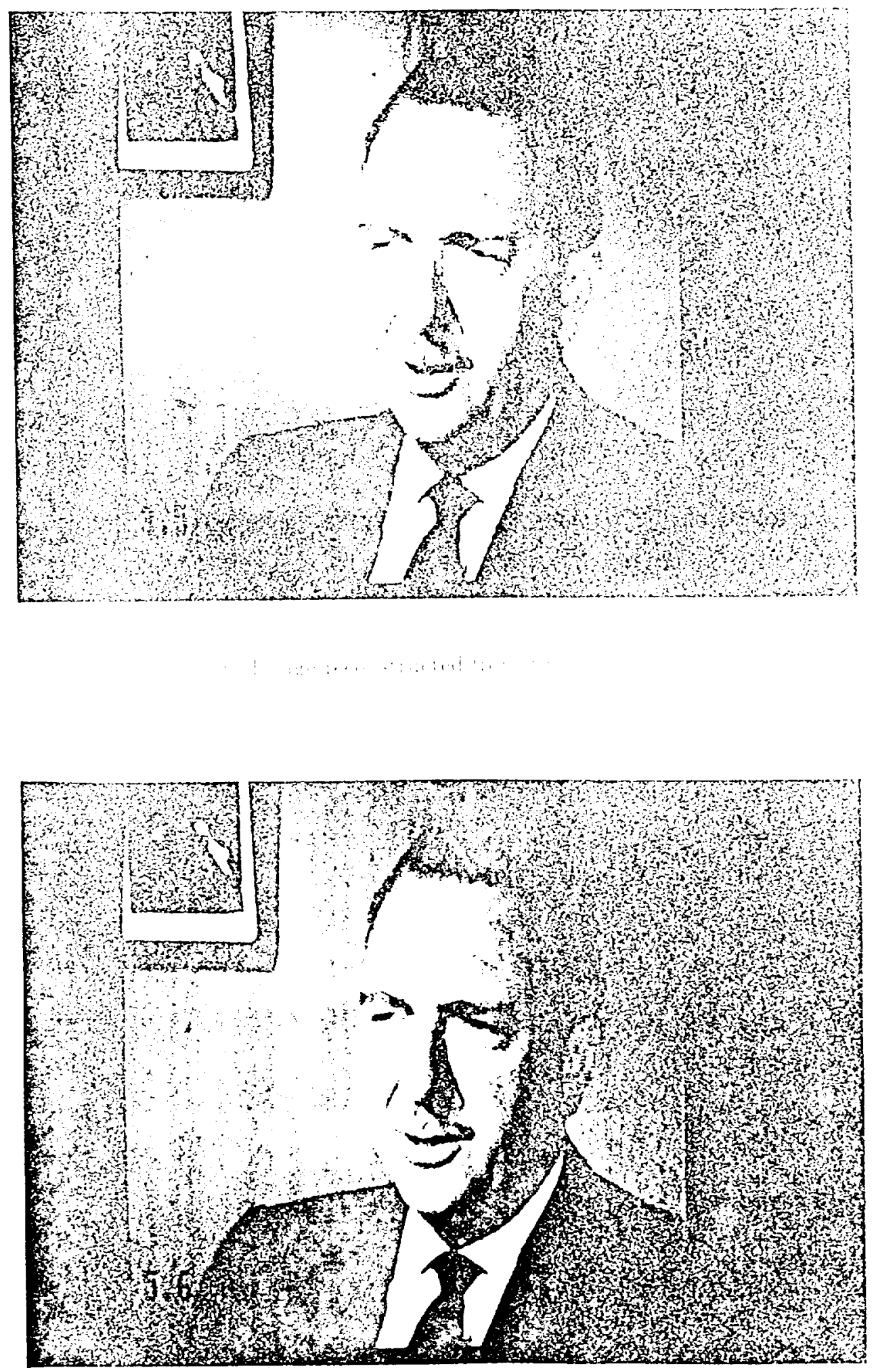


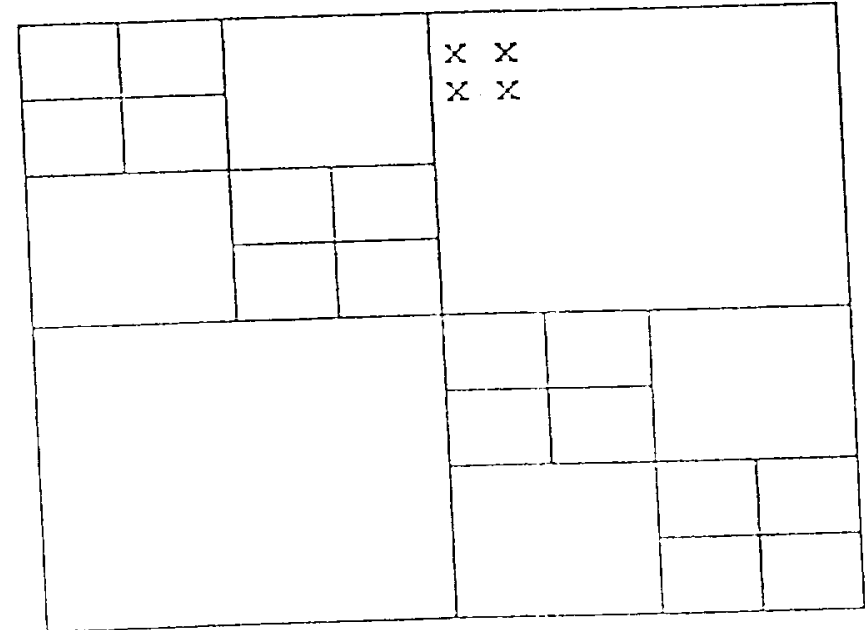

$16 \times 26$

overnead $=1,1001,1001,1001,1001,1001$

Figure 8 Overhead assigment and zonal coding. 


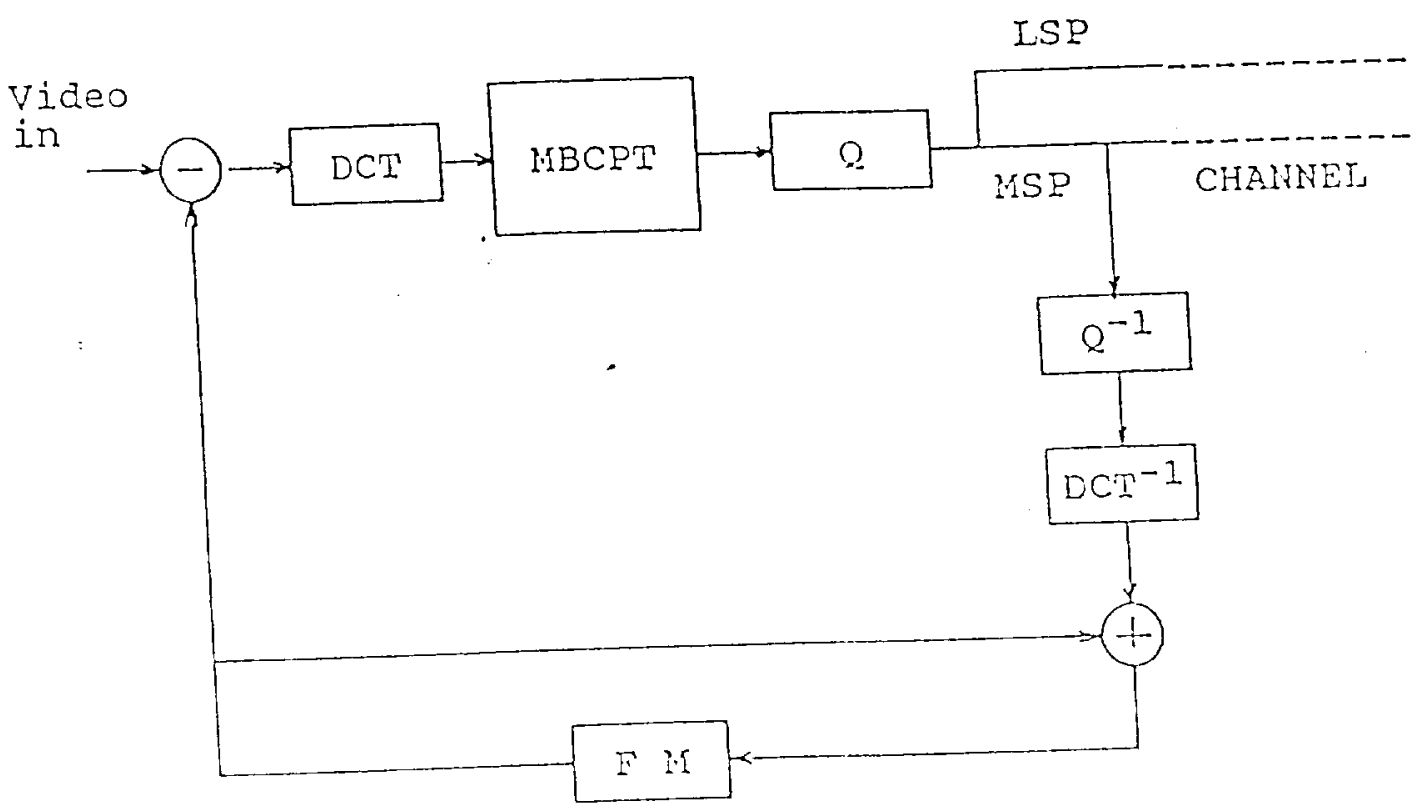

coder

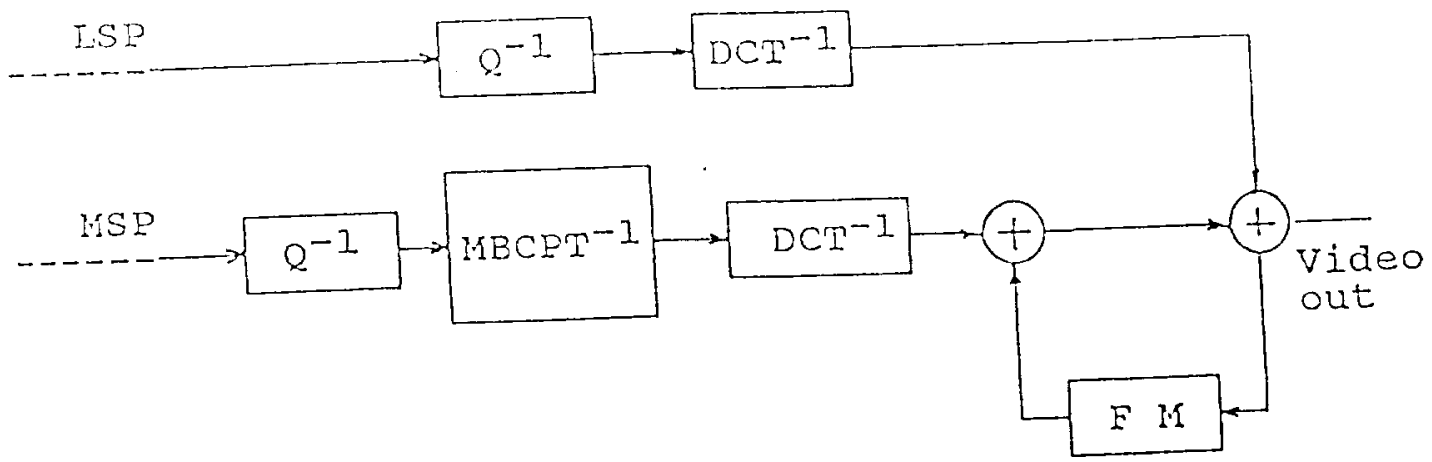

Decoder

Figure 9. Differential MBCPT coding scheme (1). 


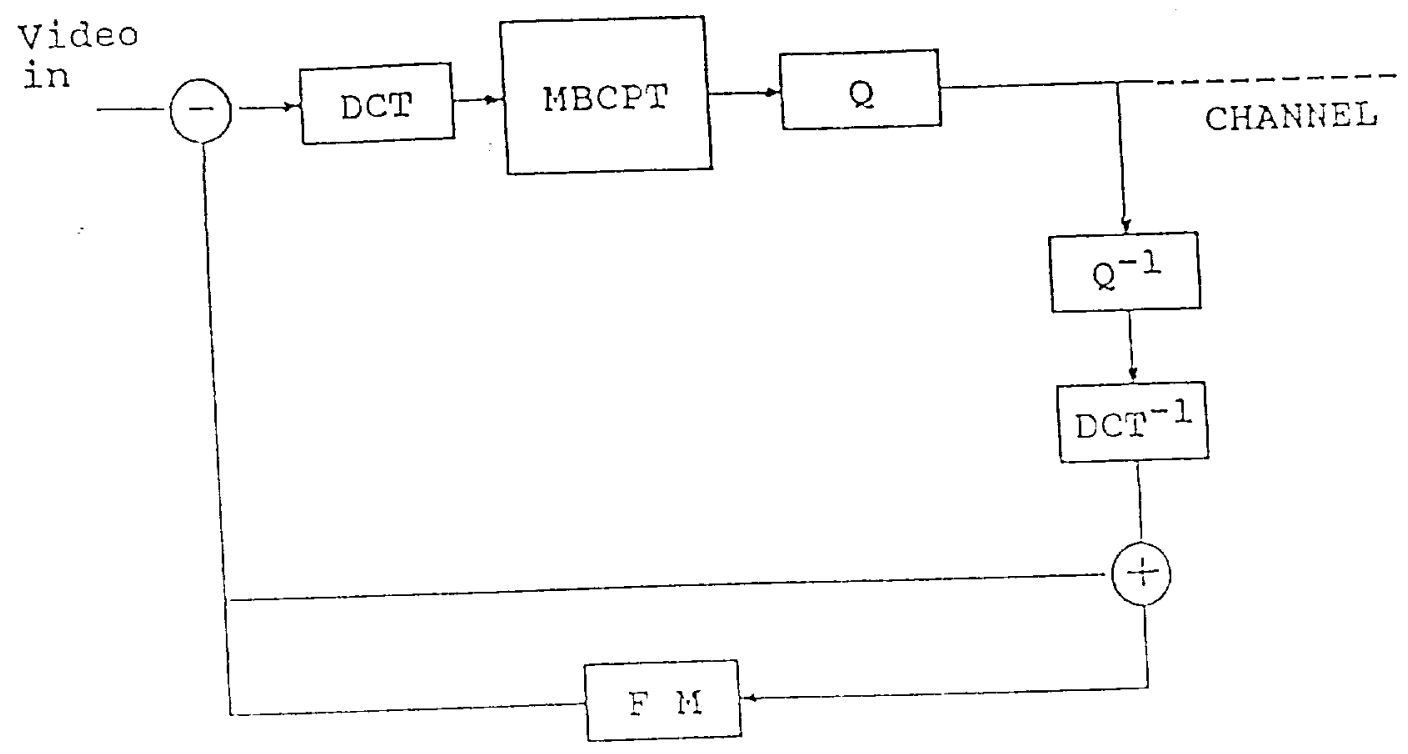

coder

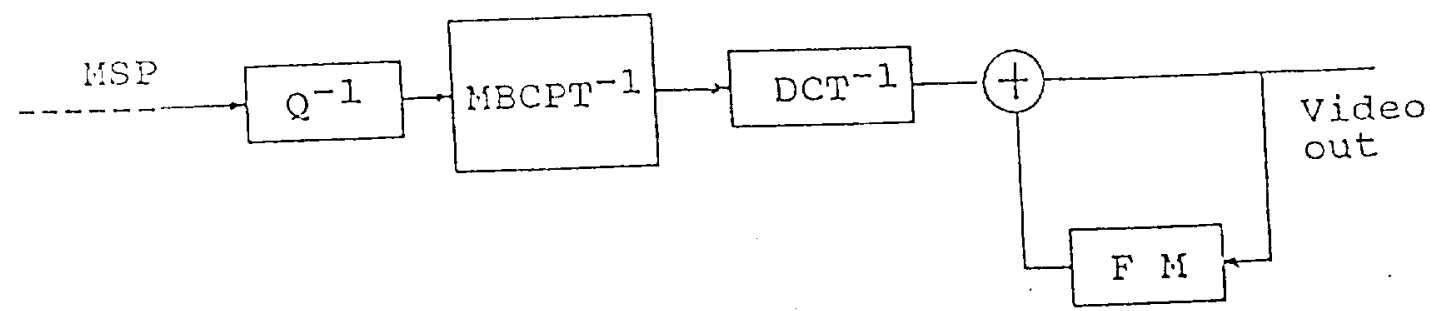

Decoder

Figure 10 Differential MBCPT coding scheme (2). 


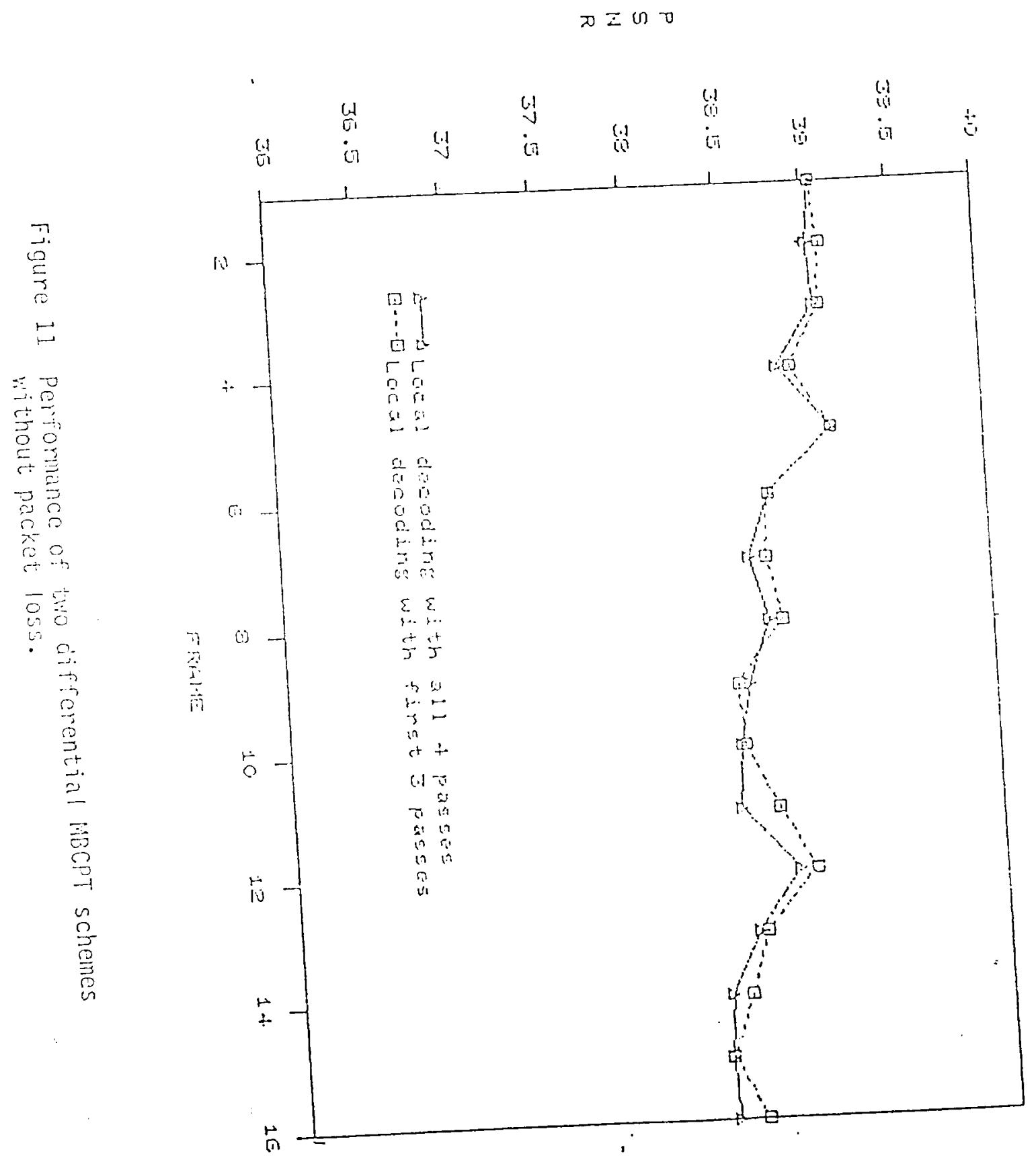




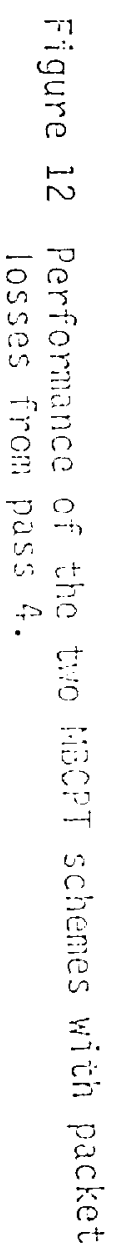

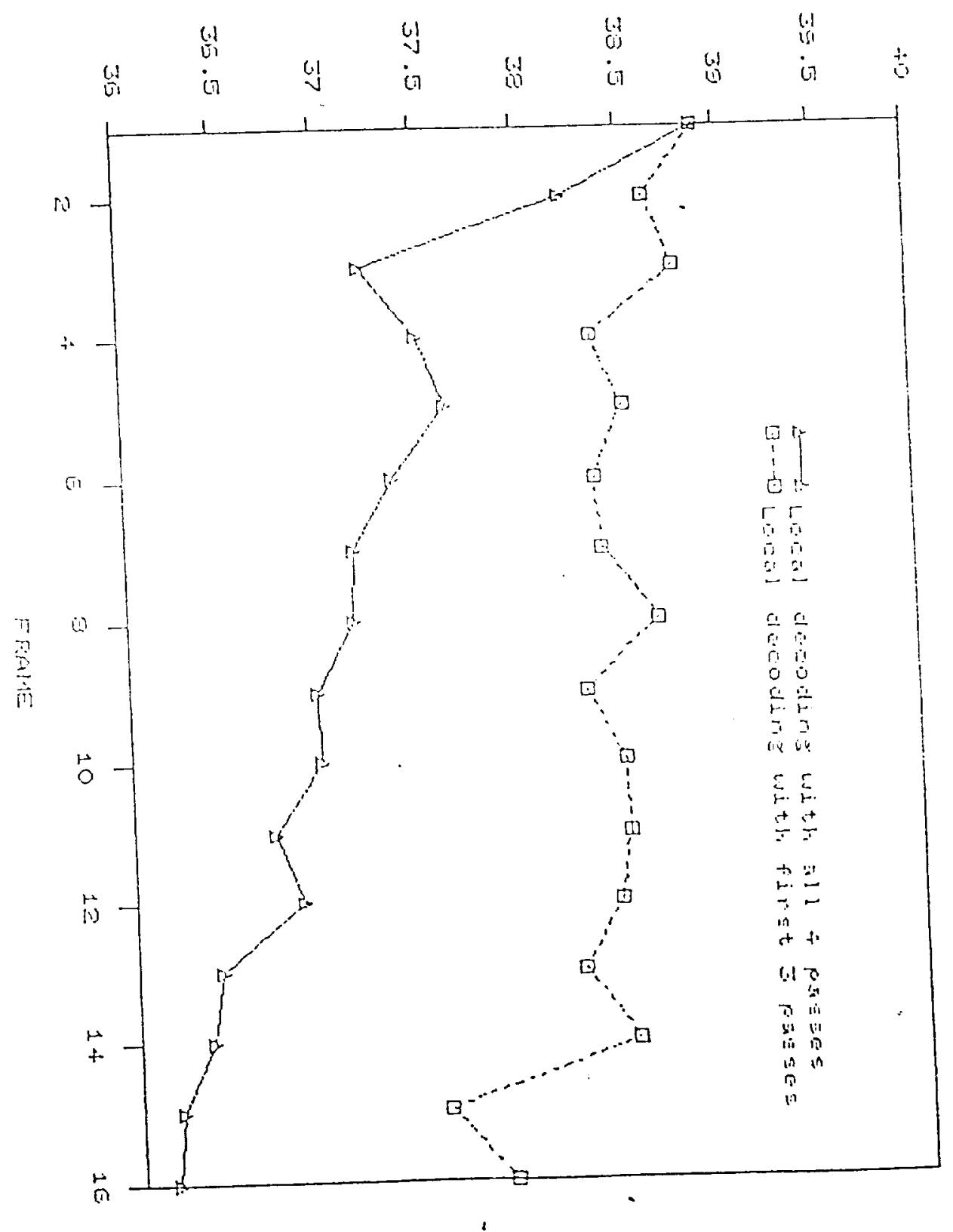




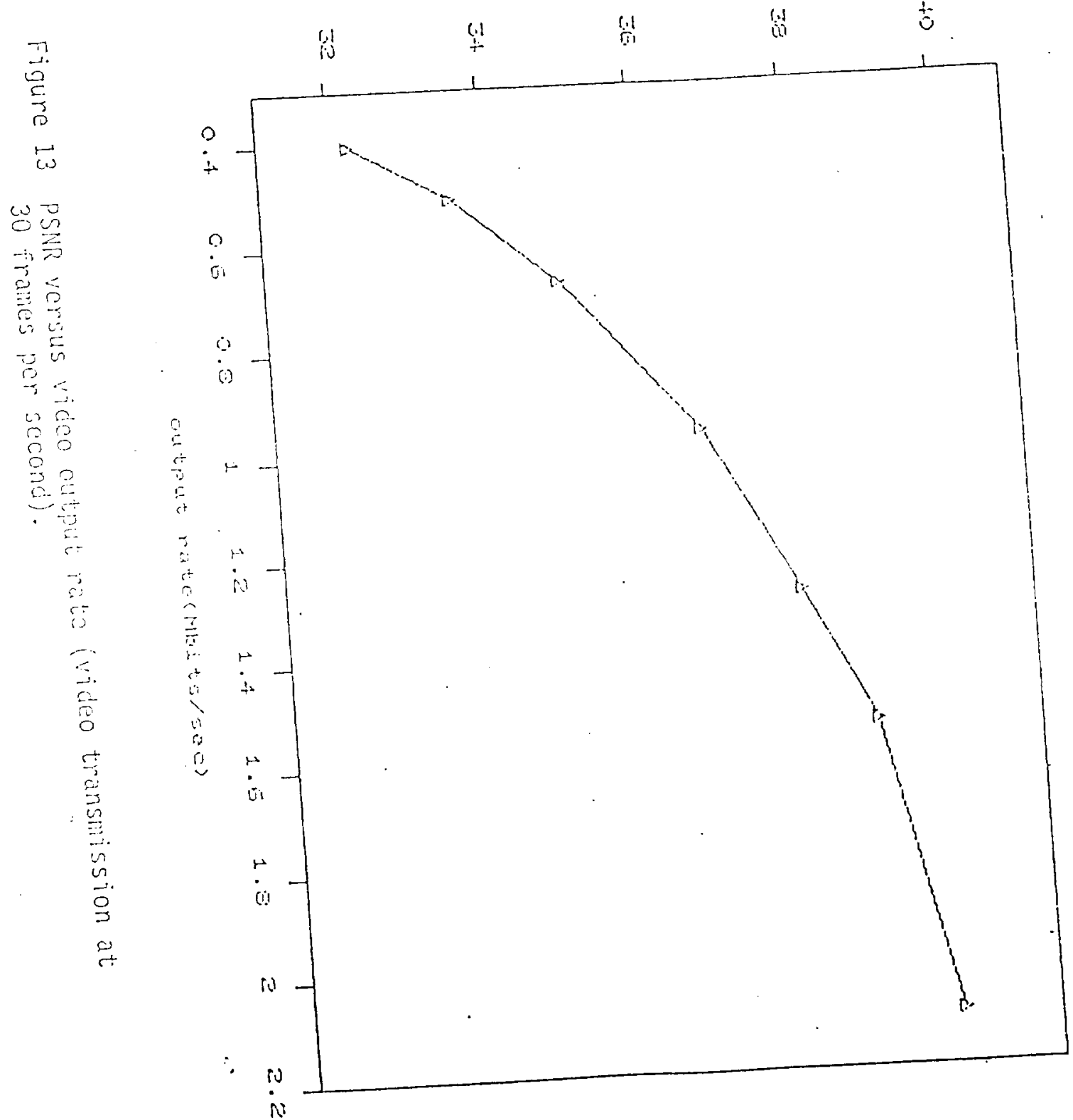


$n+b-60-5$

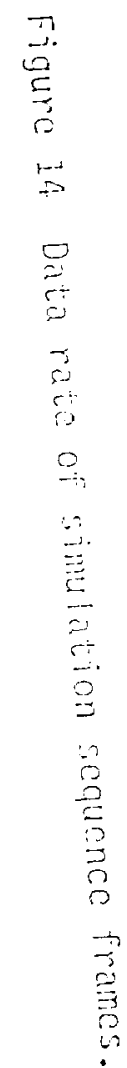

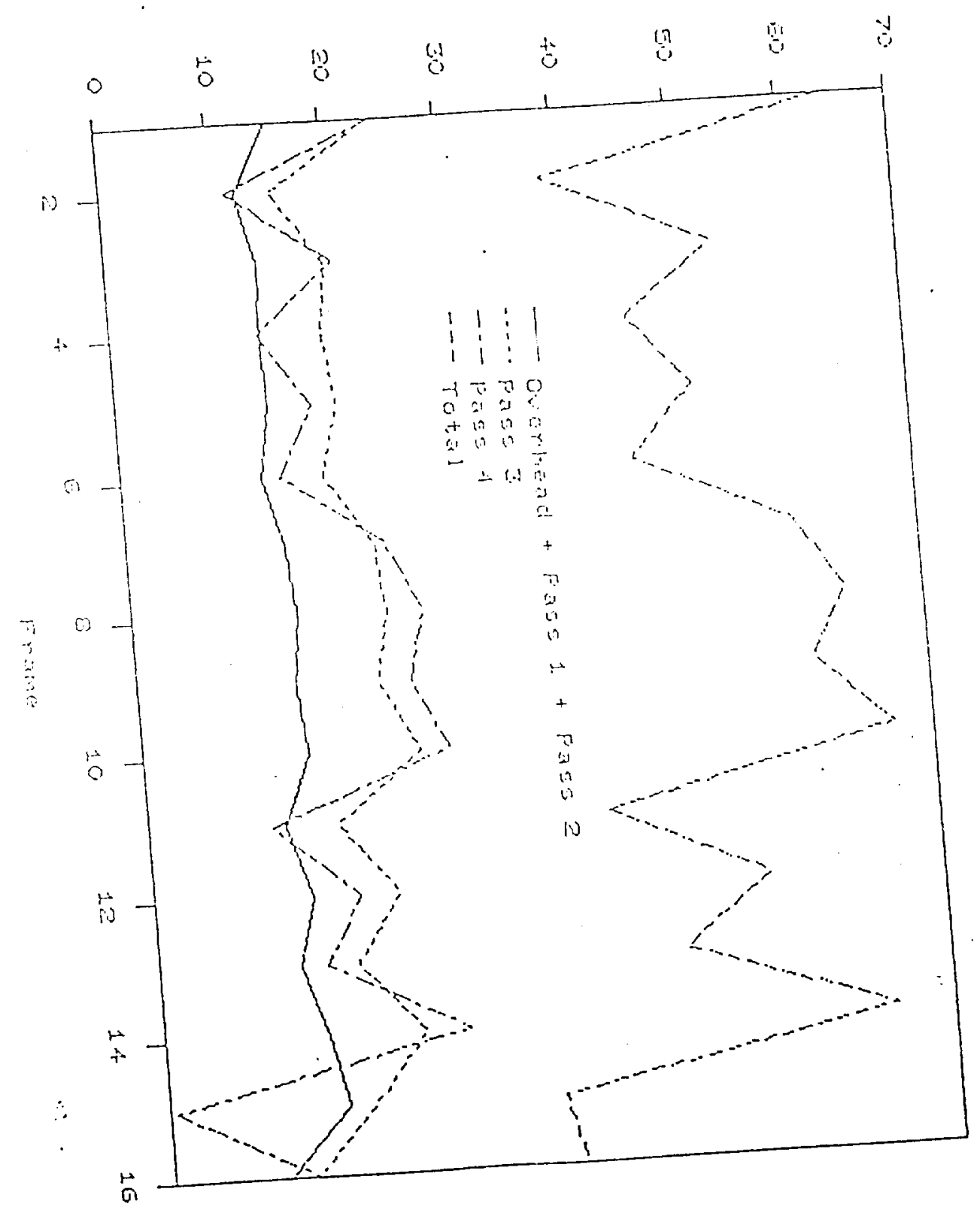


$r J u r$

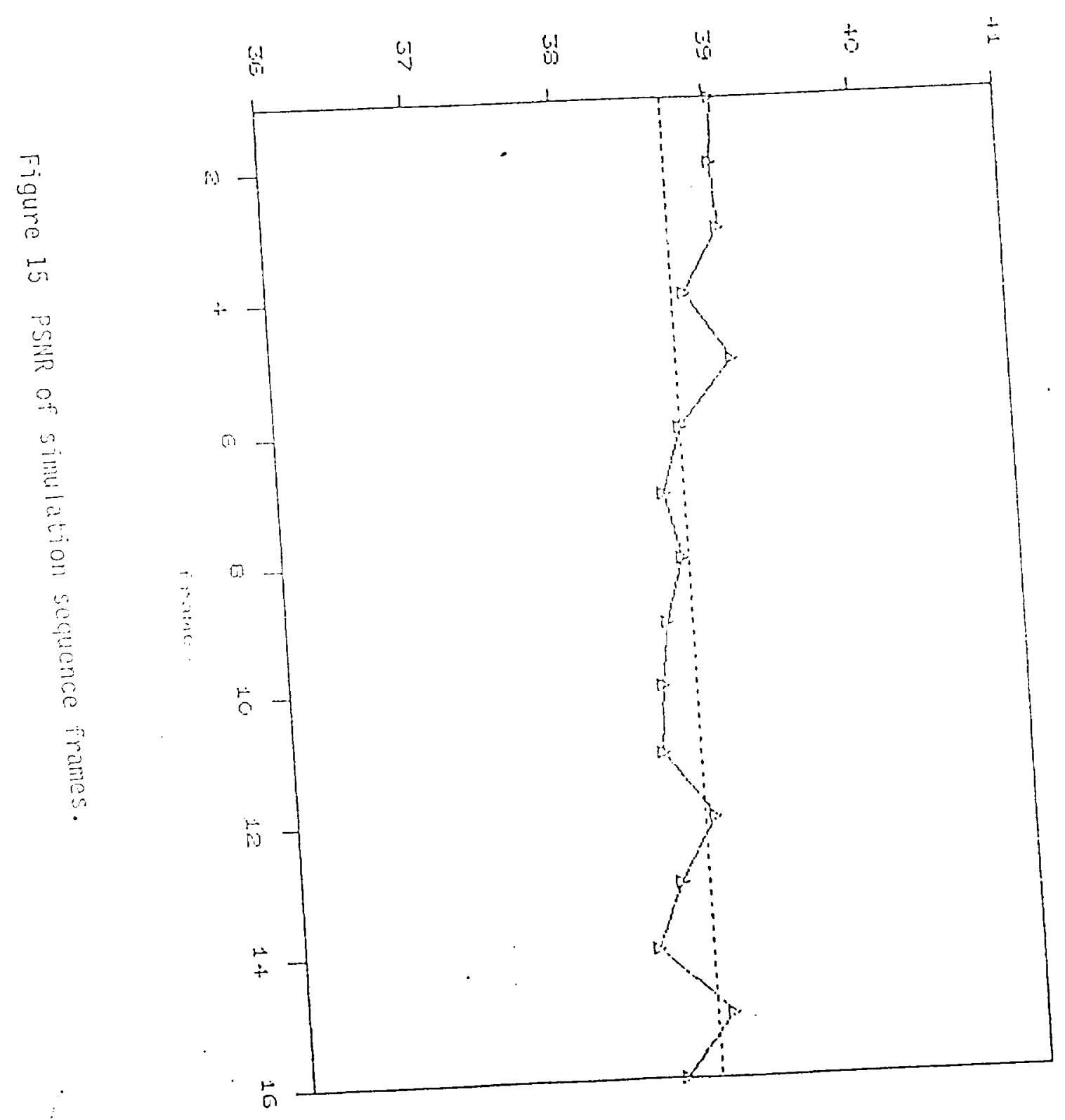



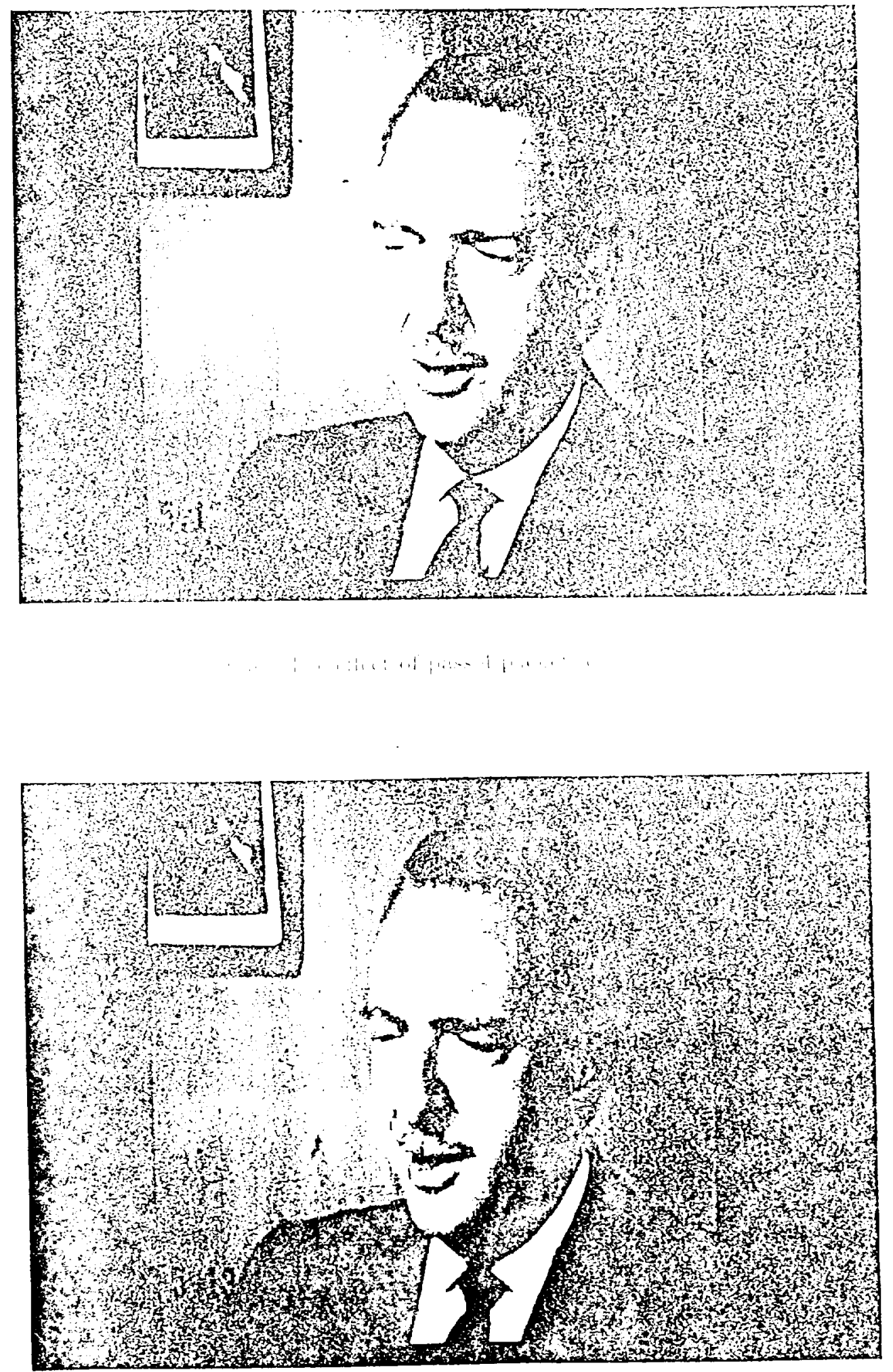

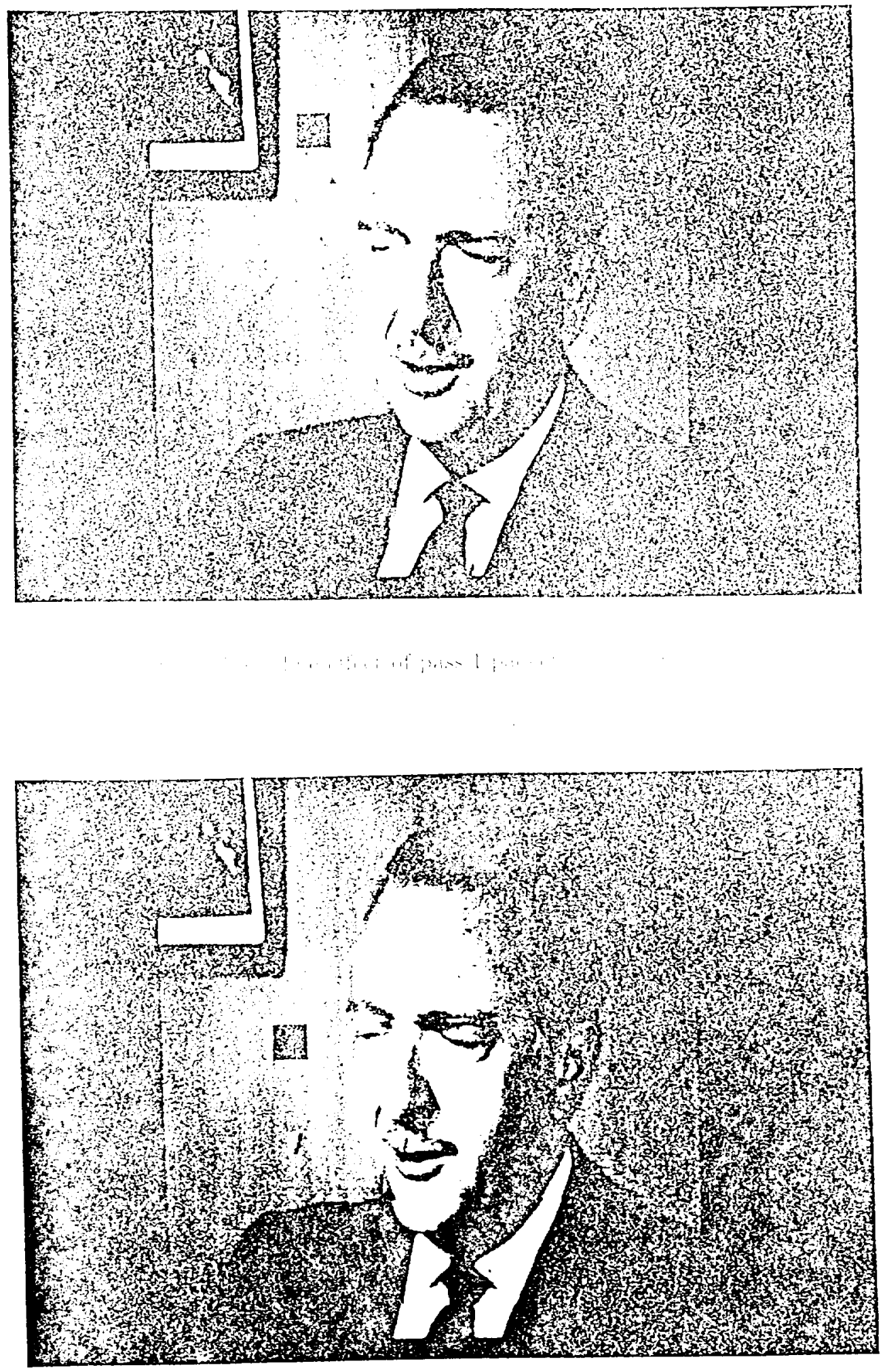
Appendix 2- Item 11 